\title{
A generalized Hölder-type inequalities for measurable operators
}

\author{
Yazhou $\operatorname{Han}^{1 *}$ (1) and Jingjing Shao ${ }^{2}$
}

${ }^{\text {*Correspondence: }}$

hyz0080@aliyun.com

${ }^{1}$ College of Mathematics and

Systems Science, Xinjiang

University, Urumqi 830046, China

Full list of author information is

available at the end of the article

\begin{abstract}
We prove a generalized Hölder-type inequality for measurable operators associated with a semi-finite von Neumann algebra which is a generalization of the result shown by Bekjan (Positivity 21:113-126, 2017). This also provides a generalization of the unitarily invariant norm inequalities for matrix due to Bhatia-Kittaneh, Horn-Mathisa, Horn-Zhan and Zou under a cohyponormal condition.
\end{abstract}

MSC: 46L51;46L52

Keywords: Measurable operator; Von Neumann algebra; Symmetric space; Hölder inequality

\section{Introduction}

Let $\mathbb{M}_{n}$ be the space of $n \times n$ complex matrices. A norm $\|\cdot\|$ on $\mathbb{M}_{n}$ is called unitarily invariant if $\|U A V\|=\|A\|$ for all $A \in \mathbb{M}_{n}$ and all unitary matrices $U, V \in \mathbb{M}_{n}$. Let $A, B \in \mathbb{M}_{n}$. In 1990, Bhatia and Kittaneh [6] established an arithmetic-geometric mean inequality for unitarily invariant norms, i.e.,

$$
\left\|A^{*} B\right\| \leq \frac{1}{2}\left\|A A^{*}+B B^{*}\right\| .
$$

Using tensor algebra techniques, a strengthening inequality of (1.1) was presented by Bhatia and Davis [5]

$$
\left\|A^{*} X B \mid\right\| \leq \frac{1}{2}\left\|A A^{*} X+X B B^{*}\right\|
$$

for $A, B, X \in \mathbb{M}_{n}$. On the other hand, let $A, B \in M_{n}$ and $r>0$, Horn and Mathisa proved in [15] the following Cauchy-Schwarz inequality for unitarily invariant norms

$$
\|\|\left|A^{*} B\right|^{r}||^{2} \leq\|\|\left(A A^{*}\right)^{r}\|\|\|\|\left(B B^{*}\right)^{r} \| .
$$

Let $A, B \in \mathbb{M}_{n}$ and $\frac{1}{p}+\frac{1}{q}=1, p, q>1, r \geq 0$. With the properties of C-S semi-norms in hand, Horn and Zhan [16] established a stronger version of inequality (1.3) as follows:

$$
\left.\|\| A^{*} B\right|^{r}\left\|\left|\leq\left\|\left(A A^{*}\right)^{\frac{r p}{2}}\right\|\right|^{\frac{1}{p}}\right\|\left(B B^{*}\right)^{\frac{r q}{2}} \|^{\frac{1}{q}} .
$$

(c) The Author(s) 2020. This article is licensed under a Creative Commons Attribution 4.0 International License, which permits use sharing, adaptation, distribution and reproduction in any medium or format, as long as you give appropriate credit to the original author(s) and the source, provide a link to the Creative Commons licence, and indicate if changes were made. The images or other third party material in this article are included in the article's Creative Commons licence, unless indicated otherwise in a credit line to the material. If material is not included in the article's Creative Commons licence and your intended use is not permitted by statutory regulation or exceeds the permitted use, you will need to obtain permission directly from the copyright holder. To view a copy of this licence, visit http://creativecommons.org/licenses/by/4.0/. 
which is the Hölder inequality for unitarily invariant norms. In particular, these authors also showed in [16] that

$$
\left\|\left.|| A^{*} X B\right|^{r}\right\|\left|\leq\left\|\left(|A|^{p} X\right)^{r}||^{\frac{1}{p}}\right\|\left(X|B|^{q}\right)^{r} \|^{\frac{1}{q}} .\right.
$$

Subsequently, a considerable different proofs, equivalent statements, along with some generalizations, refinements, and applications of inequalities (1.1)-(1.4) were discussed by many authors. We refer to $[1-3,5,15,20]$ for more information on this topic and historical references.

Let $A, B \in \mathbb{M}_{n}$ and $\frac{1}{p}+\frac{1}{q}=1, p, q>1, \alpha \in[0,1], r \geq 0$ and let $T_{X}(\alpha)=\alpha A A^{*} X+(1-$ $\alpha) X B B^{*}$. In 2015, by majorization techniques, Audenaert [2] prove an inequality that interpolates between the arithmetic-geometric mean and Cauchy-Schwarz matrix norm inequalities

$$
\left\|\left|A^{*} B\right|^{r}\right\| \leq \leq\left.\left\|\left(T_{1}(\alpha)\right)^{\frac{r p}{2}}\right\|\right|^{\frac{1}{p}}\left\|\left(T_{1}(1-\alpha)\right)^{\frac{r q}{2}}\right\| \|^{\frac{1}{q}} .
$$

Recently, Zou [20] presented the inequality for unitarily invariant norms

$$
\left\|\left.|| A^{*} X B\right|^{2 r}\right\|\left|\leq\left\|\left(T_{X}(\alpha)\right)^{r p}\right\|\right|^{\frac{1}{p}}\left\|\left(T_{X}(1-\alpha)\right)^{r q}\right\|^{\frac{1}{q}},
$$

which is a unified version of inequalities (1.1) and (1.6).

By the concept of uniform Hardy-Littlewood majorization Bekjan [8] gave a Höldertype inequality (1.4) for $\tau$-measurable operators associated with a semi-finite von Neumann algebra and for symmetric Banach spaces norm. In this paper, we will give a generalized Hölder-type inequality (1.7) for $\tau$-measurable operators under a cohyponormal condition by adopting a technique similar to the one used by Bekjan and Zou. This is a generalization of Bekjan's result in [8].

\section{Preliminaries}

Let $L_{0}$ be the set of all Lebesgue measurable functions on $(0, \infty)$. A Banach space $E \subseteq L_{0}$ with the norm $\|\cdot\|_{E}$ satisfying the condition that $f \in E$ and $\|f\|_{E} \leq\|g\|_{E}$ whenever $0 \leq f \leq$ $g, f \in L_{0}$ and $g \in F$, is said to be a Banach function space. A Banach function space $E \subseteq L_{0}$ is called a symmetric Banach function space if it follows from $f \in L_{0}, g \in E$ and $f^{*} \leq g^{*}$ that $f \in E$ and $\|f\|_{E} \leq\|g\|_{E}$, where

$$
f^{*}(t)=\inf \left\{s>0: d_{f}(s)=m\{r:|f(r)|>s\} \leq t\right\}, \quad t>0,
$$

and $m$ denotes the Lebesgue measure on $(0, \infty)$. The symmetric Banach function space $E$ is called fully if and only if $f \in E, g \in L_{0}$ and $\int_{0}^{t} f^{*}(s) d s \geq \int_{0}^{t} g^{*}(s) d s$ give us that $g \in E$ and $\|f\|_{E} \geq\|g\|_{E}$. We say that $E$ has order continuous norm if for every net $\left\{f_{i}\right\}_{i \in \Lambda} \subseteq E$ such that $f_{i} \downarrow 0$ we have $\left\|f_{i}\right\|_{E} \downarrow 0$. In particular, a symmetric Banach function space which has order continuous norm is automatically fully symmetric. For $0<r<\infty, E^{(r)}$ will denote the quasi-Banach spaces defined by

$$
E^{(r)}:=\left\{g \in L_{0}:|g|^{r} \in E\right\} \quad \text { and } \quad\|g\|_{E^{(r)}}=\left\||g|^{r}\right\|_{E}^{\frac{1}{r}} .
$$


For $r>0$, we know from [17] that if $\mathrm{E}$ is a symmetric Banach function space, then $E^{(r)}$ is a symmetric quasi-Banach space, and if $E$ has order continuous norm, then $E^{(r)}$ has order continuous norm.

We suppose that $\mathcal{M}$ is a semi-finite von Neumann algebra, namely a von Neumann algebra equipped with a semi-finite, faithful and normal trace $\tau$. We will denote by 1 the identity in $\mathcal{M}$ and $P(\mathcal{M})$ the projection lattice of $\mathcal{M}$. A closed densely defined linear operator $x$ in $\mathcal{H}$ with domain $D(x) \subseteq \mathcal{H}$ is said to be affiliated with $\mathcal{M}$ if $u^{*} x u=x$ for all unitary operators $u$ which belong to the commutant $\mathcal{M}^{\prime}$ of $\mathcal{M}$. Let $e_{s}^{\perp}(|x|)=e_{(s, \infty)}(|x|)$ be the spectral projection of $|x|$ associated with the interval $(s, \infty)$. If $x$ is affiliated with $\mathcal{M}$, $x$ will be called $\tau$-measurable if and only if $\tau\left(e_{s}^{\perp}(|x|)\right)<\infty$ for some $s>0$. The set of all $\tau$-measurable operators will be denoted by $L_{0}(\mathcal{M})$.

Definition 2.1 Let $x \in L_{0}(\mathcal{M})$ and $t>0$. The "generalized singular number of $x^{\prime \prime} \mu_{t}(x)$ is defined by

$$
\mu_{t}(x)=\inf \left\{\|x e\|: e \text { is a projection in } \mathcal{M} \text { with } \tau\left(e^{\perp}\right) \leq t\right\} .
$$

We will denote simply by $\lambda(x)$ and $\mu(x)$ the functions $t \rightarrow \lambda_{t}(x)$ and $t \rightarrow \mu_{t}(x)$, respectively. The generalized singular number function $t \rightarrow \mu_{t}(x)$ is decreasing rightcontinuous. For $x, y \in L_{0}(\mathcal{M})$ and $u, v \in \mathcal{M}$, we obtain

$$
\mu(x)=\mu(|x|)=\mu\left(x^{*}\right), \quad \mu(u x v) \leq\|u\|\|v\| \mu(x) .
$$

Moreover, let $f$ be a continuous increasing function on $[0, \infty)$ with $f(0)=0$. It follows from [11, Lemma 2.5, Lemma 2.6 and Corollary 2.8] that

$$
\mu(f(|x|))=f(\mu(|x|))
$$

and

$$
\tau(f(|x|))=\int_{0}^{\tau(1)} f\left(\mu_{t}(x)\right) d t
$$

See [11] for basic properties and detailed information on generalized singular number of $x$.

Let $E$ be a symmetric Banach function space on $(0, \infty)$. We define

$$
E(\mathcal{M})=\left\{x \in L_{0}(\mathcal{M}): \mu(x) \in E\right\} \quad \text { and } \quad\|x\|_{E(\mathcal{M})}=\|\mu(x)\|_{E}
$$

Then $\left(E(\mathcal{M}),\|\cdot\|_{E(\mathcal{M})}\right)$ is a noncommutative symmetric Banach function space. If $E=L^{p}$, then $\left(E(\mathcal{M}),\|\cdot\|_{E(\mathcal{M})}\right)$ is the usual noncommutative $L_{p}$ spaces $\left(L^{p}(\mathcal{M}),\|\cdot\|_{p}\right)$. For $0<r<\infty$, we define

$$
E(\mathcal{M})^{(r)}=\left\{x \in L_{0}(\mathcal{M}):|x|^{r} \in E(\mathcal{M})\right\} \quad \text { and } \quad\|x\|_{E(\mathcal{M})^{(r)}}=\left\||x|^{r}\right\|_{E(\mathcal{M})}^{\frac{1}{r}} .
$$

As is shown in [10, Proposition 3.1], if $E$ is a symmetric Banach function space, then $E^{(r)}(\mathcal{M})=E(\mathcal{M})^{(r)}$, where

$$
E^{(r)}(\mathcal{M})=\left\{x \in L_{0}(\mathcal{M}): \mu(x) \in E^{(r)}\right\}
$$


and $\|x\|_{E^{(r)}(\mathcal{M})}=\|\mu(x)\|_{E^{(r)}}$. It is well known that $E(\mathcal{M})^{(r)}$ is also a noncommutative fully symmetric Banach function space when $r \geq 1$ and $E$ is fully (cf. [19]).

In the following, unless stated otherwise, we will keep all previous notations throughout the paper, and we always assume that $E$ is a symmetric Banach function space on $(0, \infty)$ with order continuous norm.

\section{Main results}

We start this section with several lemmas which will be used in our proof. From [12, Theorem 3.3] and [13, Lemma 3.4] we have the following two results.

Lemma 3.1 Let $x, y \in \mathcal{M}$ and $\alpha \in[0,1]$. Then

$$
\mu_{s}\left(\left|x^{*} y\right|\right) \leq \mu_{s}\left(\alpha|x|^{\frac{1}{\alpha}}+(1-\alpha)|y|^{\frac{1}{1-\alpha}}\right) .
$$

Lemma 3.2 Let $x, y \in \mathcal{M}$ such that $x y$ is a self-adjoint operator. For every $r>0$, we obtain

$$
\int_{0}^{t} \mu_{s}(x y)^{r} d s \leq \int_{0}^{t} \mu_{s}(y x)^{r} d s, \quad t>0
$$

Remark 3.3 If $x, y$ are normal operators in $L_{0}(\mathcal{M})$, then $\mu_{s}(x y)=\mu_{s}(y x), s>0$. Indeed, we conclude from (2.1) and (2.2) (see also [11, Lemma 2.5]) that

$$
\begin{aligned}
\mu_{t}(x y) & =\mu_{t}\left(|x y|^{2}\right)^{\frac{1}{2}}=\mu_{t}\left(y^{*} x^{*} x y\right)^{\frac{1}{2}}=\mu_{t}\left(y^{*} x x^{*} y\right)^{\frac{1}{2}} \\
& =\mu_{t}\left(\left|\left(y^{*} x\right)^{*}\right|^{2}\right)^{\frac{1}{2}}=\mu_{t}\left(\left|y^{*} x\right|^{2}\right)^{\frac{1}{2}}=\mu_{t}\left(x^{*} y y^{*} x\right)^{\frac{1}{2}} \\
& =\mu_{t}\left(x^{*} y^{*} y x\right)^{\frac{1}{2}}=\mu_{t}\left(|y x|^{2}\right)^{\frac{1}{2}}=\mu_{t}(y x) .
\end{aligned}
$$

Recall that an operator $x \in L_{0}(\mathcal{M})$ is said to be hyponormal if $x^{*} x \geq x x^{*}$, cohyponormal if $x^{*}$ is hyponormal.

Lemma 3.4 Let $x, y \in \mathcal{M}$ and $r \geq 0$. If $\alpha \in[0,1]$ and $x x^{*}\left(y y^{*}\right)^{\alpha}$ is cohyponormal, then

$$
\int_{0}^{t} \mu_{s}\left(\left|x^{*} y\right|^{r}\right) d s \leq \int_{0}^{t} \mu_{s}\left(\alpha x x^{*}+(1-\alpha) y y^{*}\right)^{\frac{r}{2}} \mu_{s}\left((1-\alpha) x x^{*}+\alpha y y^{*}\right)^{\frac{r}{2}} d s, \quad t>0 .
$$

Proof By (2.2) and Lemma 3.2 we have

$$
\int_{0}^{t} \mu_{s}\left(\left|x^{*} y\right|^{r}\right) d s=\int_{0}^{t} \mu_{s}\left(y^{*} x x^{*} y\right)^{\frac{r}{2}} d s \leq \int_{0}^{t} \mu_{s}\left(x x^{*} y y^{*}\right)^{\frac{r}{2}} d s .
$$

Since $x x^{*}\left(y y^{*}\right)^{\alpha}$ is cohyponormal, [8, Corollary 4.5] yields

$$
\mu_{s}\left(x x^{*} y y^{*}\right)=\mu_{s}\left(\left[x x^{*}\left(y y^{*}\right)^{\alpha}\right]\left(y y^{*}\right)^{1-\alpha}\right) \leq \mu_{s}\left(\left(y y^{*}\right)^{\alpha} x x^{*}\left(y y^{*}\right)^{1-\alpha}\right),
$$

and hence, [11, Theorem 4.2(iii)] and Lemma 3.1 tell us that

$$
\begin{aligned}
\int_{0}^{t} \mu_{s}\left(\left|x^{*} y\right|^{r}\right) d s & \leq \int_{0}^{t} \mu_{s}\left(x x^{*} y y^{*}\right)^{\frac{r}{2}} d s \\
& =\int_{0}^{t} \mu_{s}\left(\left(y y^{*}\right)^{\alpha} x x^{*}\left(y y^{*}\right)^{1-\alpha}\right)^{\frac{r}{2}} d s
\end{aligned}
$$




$$
\begin{aligned}
& \leq \int_{0}^{t} \mu_{s}\left(\left(y y^{*}\right)^{\alpha}\left(x x^{*}\right)^{1-\alpha}\right)^{\frac{r}{2}} \mu_{s}\left(\left(x x^{*}\right)^{\alpha}\left(y y^{*}\right)^{1-\alpha}\right)^{\frac{r}{2}} d s \\
& \leq \int_{0}^{t} \mu_{s}\left((1-\alpha) x x^{*}+\alpha y y^{*}\right)^{\frac{r}{2}} \mu_{s}\left(\alpha x x^{*}+(1-\alpha) y y^{*}\right)^{\frac{r}{2}} d s .
\end{aligned}
$$

This completes the proof.

Remark 3.5 Let $x, y \in \mathcal{M}$ and $r \geq 0, \alpha \in[0,1]$. (2.1) now yields $\mu_{t}\left(x x^{*} y y^{*}\right)=\mu_{t}\left(y y^{*} x x^{*}\right)$ for all $t>0$. If $y y^{*}\left(x x^{*}\right)^{\alpha}$ is hyponormal, then from Lemma 3.4 we have

$$
\int_{0}^{t} \mu_{s}\left(\left|x^{*} y\right|^{r}\right) d s \leq \int_{0}^{t} \mu_{s}\left(\alpha x x^{*}+(1-\alpha) y y^{*}\right)^{\frac{r}{2}} \mu_{s}\left((1-\alpha) x x^{*}+\alpha y y^{*}\right)^{\frac{r}{2}} d s, \quad t>0
$$

Proposition 3.6 Let $\alpha \in[0,1], r \geq 0,1<p, q<\infty$ with $\frac{1}{p}+\frac{1}{q}=1$ and let $x, y \in E(\mathcal{M})^{(2 r)}$. If $x x^{*}\left(y y^{*}\right)^{\alpha}$ is cohyponormal, then $x^{*} y \in E(\mathcal{M})^{(r)}$,

$$
\left\|\left|x^{*} y\right|^{r}\right\|_{E(\mathcal{M})} \leq\left\|(T(\alpha))^{\frac{r p}{2}}\right\|_{E(\mathcal{M})}^{\frac{1}{p}}\left\|(T(1-\alpha))^{\frac{r q}{2}}\right\|_{E(\mathcal{M})}^{\frac{1}{q}}
$$

where $T(\alpha)=\alpha x x^{*}+(1-\alpha) y y^{*}$.

Proof If

$$
\left\|\left(\alpha x x^{*}+(1-\alpha) y y^{*}\right)^{\frac{r p}{2}}\right\|_{E(\mathcal{M})}^{\frac{1}{p}}=\infty
$$

or

$$
\left\|\left((1-\alpha) x x^{*}+\alpha y y^{*}\right)^{\frac{r q}{2}}\right\|_{E(\mathcal{M})}^{\frac{1}{q}}=\infty
$$

then the inequality (3.1) is obvious, and so we always suppose that

$$
\left\|\left(\alpha x x^{*}+(1-\alpha) y y^{*}\right)^{\frac{r p}{2}}\right\|_{E(\mathcal{M})}^{\frac{1}{p}}<\infty
$$

and

$$
\left\|\left((1-\alpha) x x^{*}+\alpha y y^{*}\right)^{\frac{r q}{2}}\right\|_{E(\mathcal{M})}^{\frac{1}{q}}<\infty .
$$

First we assume that $x, y \in E(\mathcal{M})^{(2 r)} \cap \mathcal{M}$. According to [4, Theorem 3] and Lemma 3.4, we have $x^{*} y \in E(\mathcal{M})^{(r)}$ and

$$
\begin{aligned}
\left\|\left|x^{*} y\right|^{r}\right\|_{E(\mathcal{M})} & =\left\|\mu_{s}\left(\left|x^{*} y\right|^{r}\right)\right\|_{E} \\
& \leq\left\|\mu_{s}\left(\alpha x x^{*}+(1-\alpha) y y^{*}\right)^{\frac{r}{2}} \mu_{s}\left((1-\alpha) x x^{*}+\alpha y y^{*}\right)^{\frac{r}{2}}\right\|_{E} \\
& \leq\left\|\mu_{s}\left(\alpha x x^{*}+(1-\alpha) y y^{*}\right)^{\frac{p r}{2}}\right\|_{E}^{\frac{1}{p}}\left\|\mu_{s}\left((1-\alpha) x x^{*}+\alpha y y^{*}\right)^{\frac{r q}{2}}\right\|_{E}^{\frac{1}{q}} .
\end{aligned}
$$

In the general case, for $y, x \in L_{0}(\mathcal{M})$, let $x=u|x|$ and $y=v|y|$ be the polar decomposition of $x$ and $y$, respectively. We assume also that $|y|=\int_{0}^{\infty} \lambda d e_{\lambda}(|y|)$ and $|x|=\int_{0}^{\infty} \lambda d e_{\lambda}(|x|)$ 
are the spectral decomposition of $|y|$ and $|x|$, respectively. Set $y_{n}=v \int_{0}^{n} \lambda d e_{\lambda}(|y|)$ and $x_{n}=$ $u \int_{0}^{n} \lambda d e_{\lambda}(|x|)$. Then

$$
\mu_{t}\left(x-x_{n}\right) \leq \mu_{t}(|x|) \chi_{\left(0, \tau\left(e_{[n, \infty)}(|x|)\right)\right),}, \quad\left|x-x_{n}\right|=\int_{n}^{\infty} \lambda d e_{\lambda}(|x|) .
$$

From [18, Proposition 21 of Chapter I] and [11, Lemma 3.1] we conclude that $\tau\left(e_{[n, \infty)}(|x|)\right) \rightarrow 0$ and $\mu_{t}\left(x-x_{n}\right) \downarrow 0$ as $n \rightarrow \infty$. Similarly, $\mu_{t}\left(y-y_{n}\right) \downarrow 0$ as $n \rightarrow \infty$. Since $E$ has order continuous norm, we see that

$$
\left\|\mu_{t}\left(y_{n}-y\right)^{2 r}\right\|_{E}^{\frac{1}{2}} \downarrow 0, \quad\left\|\mu_{t}\left(x_{n}-x\right)^{2 r}\right\|_{E}^{\frac{1}{2}} \downarrow 0
$$

as $n \rightarrow \infty$. Thus, [4, Theorem 3] gives

$$
\begin{aligned}
& \left\|\left|x_{n}^{*} y_{n}-x^{*} y\right|^{r}\right\|_{E(\mathcal{M})} \\
& \quad=\left\|x_{n}^{*} y_{n}-x_{n}^{*} y+x_{n}^{*} y-x^{*} y\right\|_{E(\mathcal{M})^{(r)}}^{r} \\
& \quad \leq C\left\{\left\|x_{n}^{*} y_{n}-x_{n}^{*} y\right\|_{E(\mathcal{M})^{(r)}}^{r}+\left\|x_{n}^{*} y-x^{*} y\right\|_{E(\mathcal{M})^{(r)}}^{r}\right\} \\
& \quad \leq C\left\{\left\|x_{n}^{*}\right\|_{E(\mathcal{M})^{(2 r)}}^{r}\left\|y_{n}-y\right\|_{E(\mathcal{M})^{(2 r)}}^{r}+\left\|x_{n}^{*}-x^{*}\right\|_{E(\mathcal{M})^{(r)}}^{r}\|y\|_{E(\mathcal{M})^{(r)}}^{r}\right\} \\
& \left.\quad=C\left\{\left\|\mu_{t}\left(x_{n}^{*}\right)\right\|_{E^{(2 r)}}^{r} \| \mu_{(y n}-y\right)\left\|_{E^{(2 r)}}^{r}+\right\| \mu_{t}\left(x_{n}^{*}-x^{*}\right)\left\|_{E^{(r)}}^{r}\right\| \mu_{t}(y) \|_{E^{(r)}}^{r}\right\} \\
& \quad=C\left\{\left\|\mu_{t}\left(x_{n}\right)^{2 r}\right\|_{E}^{\frac{1}{2}}\left\|\mu\left(y_{n}-y\right)^{2 r}\right\|_{E}^{\frac{1}{2}}+\left\|\mu_{t}\left(x_{n}-x\right)^{2 r}\right\|_{E}^{\frac{1}{2}}\left\|\mu_{t}(y)^{2 r}\right\|_{E}^{\frac{1}{2}}\right\},
\end{aligned}
$$

where the constant $C$ from the triangle inequality in $E(\mathcal{M})^{(r)}$. Therefore, the fact $\left\|\mu_{t}\left(x_{n}\right)^{2 r}\right\|_{E}^{\frac{1}{2}} \leq\left\|\mu_{t}(x)^{2 r}\right\|_{E}^{\frac{1}{2}}$ and (3.2) imply that $\left\|\left|x_{n}^{*} y_{n}-x^{*} y\right|^{r}\right\|_{E(\mathcal{M})} \rightarrow 0$ as $n \rightarrow \infty$. Moreover, $\left\|\left|x_{n}^{*} y_{n}\right|^{r}\right\|_{E(\mathcal{M})} \rightarrow\left\|\left|x^{*} y\right|^{r}\right\|_{E(\mathcal{M})}$ as $n \rightarrow \infty$. In the same manner we can see that

$$
\left\|\left(\alpha x_{n} x_{n}^{*}+(1-\alpha) y_{n} y_{n}^{*}\right)^{\frac{r p}{2}}\right\|_{E(\mathcal{M})}^{\frac{1}{p}} \rightarrow\left\|\left(\alpha x x^{*}+(1-\alpha) y y^{*}\right)^{\frac{r p}{2}}\right\|_{E(\mathcal{M})}^{\frac{1}{p}}
$$

and

$$
\left\|\left((1-\alpha) x_{n} x_{n}^{*}+\alpha y_{n} y_{n}^{*}\right)^{\frac{r q}{2}}\right\|_{E(\mathcal{M})}^{\frac{1}{q}} \rightarrow\left\|\left((1-\alpha) x x^{*}+\alpha y y^{*}\right)^{\frac{r q}{2}}\right\|_{E(\mathcal{M})}^{\frac{1}{q}} .
$$

This completes the proof.

Remark 3.7 Let $1<p, q<\infty$ with $\frac{1}{p}+\frac{1}{q}=1$. If $\alpha=0$, then $x x^{*}\left(y y^{*}\right)^{\alpha}=x x^{*}$ is cohyponormal. Therefore, Proposition 3.6 yields $x^{*} y \in E(\mathcal{M})^{(r)}$ and

$$
\left\|\left|x^{*} y\right|^{r}\right\|_{E(\mathcal{M})} \leq\left\|\left|y y^{*}\right|^{\frac{r p}{2}}\right\|_{E(\mathcal{M})}^{\frac{1}{p}}\left\|\left|x x^{*}\right|^{\frac{r q}{2}}\right\|_{E(\mathcal{M})}^{\frac{1}{q}},
$$

which is a main result of [4].

Remark 3.8 It is necessary for us to remark here that, it can be observed in [7, Lemma 2] without a proof that $\mu(a b)=\mu(b a)$ when $a b, b a \in L^{1}(\mathcal{M})$. However, we are not able to give it a proof at this moment. On the other hand, the authors were informed by an anonymous 
referee that $\mu(a b)=\mu(b a)$ does not hold even in the matrix case. On account of this, there could be a gap in the proof of [13, Theorem 3.6] and we give a corresponding illustration as follows: Set $r \geq 1, \alpha \in[0,1]$ and let $x x^{*}\left(y y^{*}\right)^{\alpha}$ be cohyponormal. Using Proposition 3.6 to the case $E=L_{1}$ and $p=q=2$, we have

$$
\left\|\left|x^{*} y\right|^{r}\right\|_{L^{1}(\mathcal{M})} \leq\left\|\left|\alpha x x^{*}+(1-\alpha) y y^{*}\right|^{r}\right\|_{L^{1}(\mathcal{M})}^{\frac{1}{2}}\left\|\left|(1-\alpha) x x^{*}+\alpha y y^{*}\right|^{r}\right\|_{L^{1}(\mathcal{M})^{\prime}}^{\frac{1}{2}}
$$

i.e.,

$$
\left\|x^{*} y\right\|_{L^{r}(\mathcal{M})}^{2} \leq\left\|\alpha x x^{*}+(1-\alpha) y y^{*}\right\|_{L^{r}(\mathcal{M})}\left\|(1-\alpha) x x^{*}+\alpha y y^{*}\right\|_{L^{r}(\mathcal{M})},
$$

which is the result of [14, Theorem 3.6] under a cohyponormal condition.

Theorem 3.9 Let $\alpha \in[0,1]$ and $1<p, q<\infty$ with $\frac{1}{p}+\frac{1}{q}=1$. Assume also that $r \geq$ $\max \left\{\frac{2}{p}, \frac{2}{q}\right\}, x, y \in E(\mathcal{M})^{(2 r)}$ and $z \in P(\mathcal{M})$. If $z x x^{*} z\left(z y y^{*} z\right)^{\alpha}$ is cohyponormal, then $x^{*} z y \in$ $E(\mathcal{M})^{(r)}$,

$$
\left\|\left|x^{*} z y\right|^{r}\right\|_{E(\mathcal{M})} \leq\left\|\left|T_{z}(\alpha)\right|^{\frac{r p}{2}}\right\|_{E(\mathcal{M})}^{\frac{1}{p}}\left\|\left|T_{z}(1-\alpha)\right|^{\frac{r q}{2}}\right\|_{E(\mathcal{M})}^{\frac{1}{q}},
$$

where $T_{z}(\alpha)=\alpha x x^{*} z+(1-\alpha) z y y^{*}$.

Proof Let $T(\alpha)=\alpha x x^{*}+(1-\alpha) y y^{*}$. Then $z \in P(\mathcal{M})$ and Proposition 3.6 force that

$$
\begin{aligned}
\left\|\left|x^{*} z y\right|^{r}\right\|_{E(\mathcal{M})} & =\left\|\left|x^{*} z z y\right|^{r}\right\|_{E(\mathcal{M})} \\
& \leq\left\|(z T(\alpha) z)^{\frac{r p}{2}}\right\|_{E(\mathcal{M})}^{\frac{1}{p}}\left\|(z T(1-\alpha) z)^{\frac{r q}{2}}\right\|_{E(\mathcal{M})}^{\frac{1}{q}}
\end{aligned}
$$

and

$$
2 \mu_{t}(z T(\alpha) z)=\mu(z(T(\alpha) z+z T(\alpha)) z) \leq \mu(T(\alpha) z+z T(\alpha)),
$$

and hence

$$
\left\|(z T(\alpha) z)^{\frac{r p}{2}}\right\|_{E(\mathcal{M})}^{\frac{1}{p}} \leq\left\|\left(\frac{T(\alpha) z+z T(\alpha)}{2}\right)^{\frac{r p}{2}}\right\|_{E(\mathcal{M})}^{\frac{1}{p}} .
$$

Similarly,

$$
\left\|(z T(1-\alpha) z)^{\frac{r q}{2}}\right\|_{E(\mathcal{M})}^{\frac{1}{q}} \leq\left\|\left|\frac{z T(1-\alpha)+T(1-\alpha) z}{2}\right|^{\frac{r q}{2}}\right\|_{E(\mathcal{M})}^{\frac{1}{q}} .
$$

Therefore,

$$
\begin{aligned}
& \left\|\left|x^{*} z y\right|^{r}\right\|_{E(\mathcal{M})} \\
& \quad \leq\left\|\left|\frac{z T(\alpha)+T(\alpha) z}{2}\right|^{\frac{r p}{2}}\right\|_{E(\mathcal{M})}^{\frac{1}{p}}\left\|\left|\frac{z T(1-\alpha)+T(1-\alpha) z}{2}\right|^{\frac{r q}{2}}\right\|_{E(\mathcal{M})}^{\frac{1}{q}} .
\end{aligned}
$$


A simple computation shows

$$
\frac{T(\alpha) z+z T(\alpha)}{2}=\frac{1}{2}\left\{\alpha x x^{*} z+(1-\alpha) z y y^{*}+\left(\alpha x x^{*} z+(1-\alpha) z y y^{*}\right)^{*}\right\} .
$$

According to [11, Theorem 4.4(ii)] and (2.1), we have

$$
\begin{aligned}
\int_{0}^{t} & \mu_{s}\left(\frac{T(\alpha) z+z T(\alpha)}{2}\right) d s \\
\leq & \int_{0}^{t} \mu_{s}\left(\frac{1}{2}\left(\alpha x x^{*} z+(1-\alpha) z y y^{*}\right)\right) d s \\
& +\int_{0}^{t} \mu_{s}\left(\frac{1}{2}\left(\alpha x x^{*} z+(1-\alpha) z y y^{*}\right)^{*}\right) d s \\
= & \int_{0}^{t} \mu_{s}\left(\alpha x x^{*} z+(1-\alpha) z y y^{*}\right) d s .
\end{aligned}
$$

Since $\frac{r p}{2} \geq 1$, from [9, Theorem 2.1] and (2.2) we can assert that

$$
\begin{aligned}
\int_{0}^{t} \mu_{s}\left(\left|\frac{T(\alpha) z+z T(\alpha)}{2}\right|^{\frac{r p}{2}}\right) d s & =\int_{0}^{t} \mu_{s}\left(\frac{T(\alpha) z+z T(\alpha)}{2}\right)^{\frac{r p}{2}} d s \\
& \leq \int_{0}^{t} \mu_{s}\left(\alpha x x^{*} z+(1-\alpha) z y y^{*}\right)^{\frac{r p}{2}} d s \\
& =\int_{0}^{t} \mu_{s}\left(\left|\alpha x x^{*} z+(1-\alpha) z y y^{*}\right|^{\frac{r p}{2}}\right) d s .
\end{aligned}
$$

Consequently,

$$
\left\|\left|\frac{z T(\alpha)+T(\alpha) z}{2}\right|^{\frac{r p}{2}}\right\|_{E(\mathcal{M})} \leq\left\|\left|\alpha x x^{*} z+(1-\alpha) z y y^{*}\right|^{\frac{r p}{2}}\right\|_{E(\mathcal{M})}
$$

In the same way as used above, we can also prove that

$$
\left\|\left|\frac{z T(1-\alpha)+T(1-\alpha) z}{2}\right|^{\frac{r q}{2}}\right\|_{E(\mathcal{M})} \leq\left\|\left|(1-\alpha) x x^{*} z+\alpha z y y^{*}\right|^{\frac{r q}{2}}\right\|_{E(\mathcal{M})} .
$$

Therefore, inequalities (3.4), (3.5) and (3.6) give

$$
\left\|\left|x^{*} z y\right|^{r}\right\|_{E(\mathcal{M})} \leq\left\|\left|\alpha x x^{*} z+(1-\alpha) z y y^{*}\right|^{\frac{r p}{2}}\right\|_{E(\mathcal{M})}^{\frac{1}{p}}\left\|\left|(1-\alpha) x x^{*} z+\alpha z y y^{*}\right|^{\frac{r q}{2}}\right\|_{E(\mathcal{M})}^{\frac{1}{q}}
$$

Remark 3.10 Let $\alpha \in[0,1]$ and $1<p, q<\infty$ with $\frac{1}{p}+\frac{1}{q}=1$. Assume also that $r \geq \max \left\{\frac{2}{p}, \frac{2}{q}\right\}$, $x, y \in E(\mathcal{M})^{(2 r)}$ and $z \in \mathcal{M}$. We write $T_{z}(\alpha)=\alpha x x^{*} z+(1-\alpha) z y y^{*}$ and we wish to prove

$$
\left\|\left|x^{*} z y\right|^{r}\right\|_{E(\mathcal{M})} \leq\left\|\left|T_{z}(\alpha)\right|^{\frac{r p}{2}}\right\|_{E(\mathcal{M})}^{\frac{1}{p}}\left\|\left|T_{z}(1-\alpha)\right|^{\frac{r q}{2}}\right\|_{E(\mathcal{M})}^{\frac{1}{q}}
$$

However, we do not succeed in proving it at this moment. 
Theorem 3.11 Let $r>0$ and $x, y \in E(\mathcal{M})^{(2 r)}, 0 \leq z \in \mathcal{M}$. Assume also that $\alpha \in[0,1]$ and $1<p, q<\infty$ with $\frac{1}{p}+\frac{1}{q}=1$. If $z^{\frac{1}{2}} x x^{*} z^{\frac{1}{2}}\left(z^{\frac{1}{2}} y y^{*} z^{\frac{1}{2}}\right)^{\alpha}$ is cohyponormal, then $x^{*} z y \in E(\mathcal{M})^{(r)}$ and

$$
\left\|\left|x^{*} z y\right|^{r}\right\|_{E(\mathcal{M})} \leq\left\||T(\alpha) z|^{\frac{r p}{2}}\right\|_{E(\mathcal{M})}^{\frac{1}{p}}\left\||T(1-\alpha) z|^{\frac{r q}{2}}\right\|_{E(\mathcal{M})}^{\frac{1}{q}}
$$

where $T(\alpha)=\alpha x x^{*}+(1-\alpha) y y^{*}$.

Proof First it follows from [4, Theorem 3] that $x^{*} z y \in E(\mathcal{M})^{(r)}$. Since $z$ is positive, Proposition 3.6 gives

$$
\begin{aligned}
\left\|\left|x^{*} z y\right|^{r}\right\|_{E(\mathcal{M})} & =\left\|\left|x^{*} z^{\frac{1}{2}} z^{\frac{1}{2}} y\right|^{r}\right\|_{E(\mathcal{M})} \\
& \leq\left\|\left(z^{\frac{1}{2}} T(\alpha) z^{\frac{1}{2}}\right)^{\frac{r p}{2}}\right\|_{E(\mathcal{M})}^{\frac{1}{p}}\left\|\left(z^{\frac{1}{2}} T(1-\alpha) z^{\frac{1}{2}}\right)^{\frac{r q}{2}}\right\|_{E(\mathcal{M})}^{\frac{1}{q}},
\end{aligned}
$$

and hence Lemma 3.2 leads to

$$
\begin{aligned}
\left\|\left(z^{\frac{1}{2}} T(\alpha) z^{\frac{1}{2}}\right)^{\frac{r p}{2}}\right\|_{E(\mathcal{M})}^{\frac{1}{p}} & =\left\|z^{\frac{1}{2}} T(\alpha) z^{\frac{1}{2}}\right\|_{E(\mathcal{M})^{\left(\frac{r p}{2}\right)}}^{\frac{r}{2}} \\
& \leq\|T(\alpha) z\|_{\left.E(\mathcal{M})^{\frac{r p}{2}}\right)}^{\frac{r}{2}}=\left\|\left.T(\alpha) z\right|^{\frac{r p}{2}}\right\|_{E(\mathcal{M})}^{\frac{1}{p}} .
\end{aligned}
$$

Similarly,

$$
\left\|\left(z^{\frac{1}{2}} T(1-\alpha) z^{\frac{1}{2}}\right)^{\frac{r q}{2}}\right\|_{E(\mathcal{M})}^{\frac{1}{q}} \leq\left\||T(1-\alpha) z|^{\frac{r q}{2}}\right\|_{E(\mathcal{M})}^{\frac{1}{q}} .
$$

Therefore,

$$
\left\|\left|x^{*} z y\right|^{r}\right\|_{E(\mathcal{M})} \leq\left\||T(\alpha) z|^{\frac{r p}{2}}\right\|_{E(\mathcal{M})}^{\frac{1}{p}}\left\||T(1-\alpha) z|^{\frac{r q}{2}}\right\|_{E(\mathcal{M})}^{\frac{1}{q}} .
$$

This completes the proof.

Remark 3.12 (1) Let $\alpha \in[0,1]$ and $1<p, q<\infty$ with $\frac{1}{p}+\frac{1}{q}=1$ and let $r \geq \max \left\{\frac{2}{p}\right.$, $\left.\frac{2}{q}\right\}$. For $x, y \in E(\mathcal{M})^{(2 r)}$ and $z \in P(\mathcal{M})$, write $T_{z}(\alpha)=\alpha x x^{*} z+(1-\alpha) z y y^{*}$ and $T(\alpha)=\alpha x x^{*}+(1-$ $\alpha) y y^{*}$. Assume also that $z x x^{*} z\left(z y y^{*} z\right)^{\alpha}$ is cohyponormal. Combining Theorem 3.11 with Theorem 3.9 we have

$$
\left\|\left|x^{*} z y\right|^{r}\right\|_{E(\mathcal{M})} \leq \min \{a, b\},
$$

where

$$
a=\left\|\left|T_{z}(\alpha)\right|^{\frac{r p}{2}}\right\|_{E(\mathcal{M})}^{\frac{1}{p}}\left\|\left|T_{z}(1-\alpha)\right|^{\frac{r q}{2}}\right\|_{E(\mathcal{M})}^{\frac{1}{q}}
$$

and

$$
b=\left\||T(\alpha) z|^{\frac{r p}{2}}\right\|_{E(\mathcal{M})}^{\frac{1}{p}}\left\||T(1-\alpha) z|^{\frac{r q}{2}}\right\|_{E(\mathcal{M})}^{\frac{1}{q}} .
$$


(2) Let $r>0, x, y \in E(\mathcal{M})^{(2 r)}, 0 \leq z \in \mathcal{M}$ and $\alpha \in[0,1], 1<p, q<\infty$ with $\frac{1}{p}+\frac{1}{q}=1$. If $z^{\frac{1}{2}} y y^{*} z^{\frac{1}{2}}\left(z^{\frac{1}{2}} x x^{*} z^{\frac{1}{2}}\right)^{\alpha}$ is cohyponormal, then $x^{*} z y \in E(\mathcal{M})^{(r)}$. Moreover, the fact $\mu_{t}\left(\left|x^{*} z y\right|^{r}\right)=$ $\mu_{t}\left(x^{*} z y\right)^{r}=\mu_{t}\left(y^{*} z x\right)^{r}=\mu_{t}\left(\left|y^{*} z x\right|^{r}\right)$ and Theorem 3.11 yields

$$
\left\|\left|y^{*} z x\right|^{r}\right\|_{E(\mathcal{M})} \leq\left\||T(\alpha) z|^{\frac{r p}{2}}\right\|_{E(\mathcal{M})}^{\frac{1}{p}}\left\||T(1-\alpha) z|^{\frac{r q}{2}}\right\|_{E(\mathcal{M})}^{\frac{1}{q}}
$$

where $T(\alpha)=\alpha x x^{*}+(1-\alpha) y y^{*}$.

\section{Acknowledgements}

The authors is grateful to the referee for very useful comments.

\section{Funding}

This research is partially supported by Tianshan youth project No. 2018Q012 and the National Natural Science Foundation of China No. 11761067 and No. 11701255.

\section{Availability of data and materials}

We declare that the materials described in the manuscript, including all relevant raw data, will be freely available to any scientist wishing to use them for non-commercial purposes, without breaching participant confidentiality.

\section{Competing interests}

The author declares that there is no conflict of interests regarding the publication of this paper.

\section{Authors' contributions}

Both authors contributed equally and significantly in writing this article. Both authors read and approved the final manuscript.

\section{Author details}

${ }^{1}$ College of Mathematics and Systems Science, Xinjiang University, Urumqi 830046, China. ${ }^{2}$ School of Mathematics and Statistics Sciences, Ludong University, Yantai, 264000, China.

\section{Publisher's Note}

Springer Nature remains neutral with regard to jurisdictional claims in published maps and institutional affiliations.

Received: 2 November 2019 Accepted: 30 July 2020 Published online: 08 August 2020

\section{References}

1. Al-Natoor, A., Hirzallah, O., Kittaneh, F.: Interpolating inequalities for functions of positive semidefinite matrices. Banach J. Math. Anal. 12, 955-969 (2018)

2. Audenaert, K.M.R.: Interpolating between the arithmetic-geometric mean and Cauchy-Schwarz matrix norm inequalities. Oper. Matrices 9, 475-479 (2015)

3. Bakherad, M., Lashkaripour, R., Hajmohamadi, M.: Extensions of interpolation between the arithmetic-geometric mean inequality for matrices. J. Inequal. Appl. 2017, 209 (2017)

4. Bekjan, T.N., Ospanov, M.N.: Hölder-type inequalities of measurable operators. Positivity 21, 113-126 (2017)

5. Bhatia, R., Davis, C.: More matrix forms of the arithmetic-geometric mean inequality. SIAM J. Matrix Anal. Appl. 14 132-136 (1993)

6. Bhatia, R., Kittaneh, F.: On the singular values of a product of operators. SIAM J. Matrix Anal. Appl. 11, 272-277 (1990)

7. Bikchentaev, A.: Majorization for products of measurable operators. Int. J. Theor. Phys. 37, 571-576 (1998)

8. Bikchentaev, A.M.: On $\tau$-compactness of products of measurable operators. Int. J. Theor. Phys. 56, 3819-3830 (2017)

9. Chong, K.M.: Some extensions of a theorem of Hardy, Littlewood and Pólya and their applications. Can. J. Math. 26, $1321-1340$ (1974)

10. Dodds, P.G., Dodds, T.K., Sukochev, F.A.: On p-convexity and q-concavity in noncommutative symmetric spaces. Integral Equ. Oper. Theory 78, 91-114 (2014)

11. Fack, T., Kosaki, H.: Generalized s-numbers of $\tau$-measurable operators. Pac. J. Math. 123, 269-300 (1986)

12. Farenick, D.R., Manjegani, S.M.: Young's inequality in operator. J. Ramanujan Math. Soc. 20, 107-124 (2005)

13. Han, Y.: Submajorization and $p$-norm inequalities associated with $\tau$-measurable operators. Linear Multilinear Algebra 65, 2199-2211 (2017)

14. Han, Y., Shao, J.: Notes on two recent results of Audenaert. Electron. J. Linear Algebra 31, 147-155 (2016)

15. Horn, R.A., Mathisa, R.: Cauchy-Schwarz inequalities associated with positive semidefinite matrices. Linear Algebra Appl. 142, 63-82 (1990)

16. Horn, R.A., Zhan, X.: Inequalities for C-S seminorms and Lieb functions. Linear Algebra Appl. 291, 103-113 (1999)

17. Lindenstrauss, J., Tzafriri, L.: Classical Banach Spaces II: Function Spaces. Ergeb. Math. Grenzgeb. (Results in Mathematics and Related Areas), vol. 97. Springer, Berlin (1979)

18. Terp, M.: $L^{p}$ spaces associated with von Neumann algebras, Notes, Copenhagen Univ. (1981)

19. Xu, Q.: Analytic functions with values in lattices and symmetric spaces of measurable operators. Math. Proc. Camb. Philos. Soc. 109, 541-563 (1991)

20. Zou, L.: Unification of the arithmetic-geometric mean and Höder inequalities for unitarily invariant norms. Linear Algebra Appl. 562, 154-162 (2019) 